\title{
An Efficient Noisy Pixels Detection Model for CT Images using Extreme Learning Machines
}

\author{
Abidin ÇALIŞKAN, Ulus ÇEVIK
}

\begin{abstract}
In this study, a new and rapid hidden resource decomposition method has been proposed to determine noisy pixels by adopting the extreme learning machines (ELM) method. The goal of this method is not only to determine noisy pixels, but also to protect critical structural information that can be used for disease diagnosis. In order to facilitate the diagnosis and also the treatment of patients in medicine, two-dimensional (2-D) images were calculated tomography (CT) which is obtained using medical imaging techniques. Utilizing a large number of CT images, promising results have been obtained from these experiments. The proposed method has shown a significant improvement on mean squared error and peak signal-to-noise ratio. The experimental results indicate that the proposed method is statistically efficient, and it has a good performance with a high learning speed. In the experiments, the results demonstrated that remarkable successive rates were obtained through the ELM method.
\end{abstract}

Keywords: detection; ELM; filtering; medical imaging; MSE; PSNR

\section{INTRODUCTION}

At present, computer graphics are being used for medical imaging and many other operations [1]. Medical imaging is a crucial part of healthcare for the identification of diseases. There are various imaging techniques for different applications to observe the anatomical and physiological conditions of the patient [2]; however, these techniques can produce noise during the medical image processing. Clear CT or magnetic resonance (MR) images are significantly important for the analysis of the disease. If the noise and artefacts are not sufficiently minimized, diagnosis becomes very difficult. Visualization plays an efficient role in medical domains, medical education, and diagnosis of the diseases, without requiring surgical intervention. Especially, simulation in surgery is an important factor that accelerates technological development. Generally, the images obtained from medical processes can be repaired before using. The repairing process involves the denoising of the image, adjustments of contrast, and brightness, and removal of the artificial figures from the entire image or a part of it. Such artificial figures are objects that are placed in the body, such as a stopping or prosthesis [3]. Sometimes, these artefacts can have adverse effects. Yan et al, proposed a new system to remove unnecessary regions in the image using a two-layer filtering mechanism [4]. The noises need to be removed in order to attain a clear image.

Many filtering methods are present for the process of denoising [5-7]. Coupé et al. [8] presented a new denoising method for 2-D image, which was initially proposed by Buades et al. [9]. They proposed to improve the non-local means filter (NLMF) with an automatic tuning of the filtering parameter. Alternatively, this method accepts that images contain high self-similarity. Efros and Leung [10] first introduced the concept of selfsimilarity for texture synthesis. Weijer and Boomgaard [11] reduced the normally distributed noise without blurring the edges and utilized the theoretical foundation for local mode filtering. Consequently, the filter could easily be extended to multi-channel imaging. They proposed the use of the Gaussian filter for the utilization of the local mode.

In order to effectively achieve noise reduction on medical images, Suganthi and Deepa [12] conducted an investigation. In their study, a contrast enhancement and noise reduction technique was applied. In the system, the medical image was provided as an input, and the image was denoised using various filters. The quality and accuracy of the study has been measured using the peak signal-to-noise ratio (PSNR) and mean squared error (MSE) values, and the filtered images are evaluated in accordance with the obtained values. Kalavathi and Priya [13] have proposed a method known as histogrambased localized wiener filter (HLWF) for the noise reduction of MR brain images. Bhadauria and Singh [14] applied an edge detection algorithm based on wavelet transform and canny operator. They adopted the PSNR method to evaluate the success of the method in effectively denoising. Gabralla et al. [15] presented a new denoising scheme applying the soft-thresholding method and modifying the wavelet coefficients. Chen et al. [16] utilized multiple repetitive structures to denoise an image. Their study, which focused on finding similar structures in an image, is known as the collaborative non-local means (CNLM). Yang et al. [17] suggested a framework for pre-smoothing non-local means (PSNLM) filtering with image transformation. Jifara et al. [18] modelled an effective deep feed forward convolutional neural network (CNN) utilizing a small training data set to denoise medical images. Yang et al. [19] designed a perceptive deep CNN based on a perceptual loss as an objective function to denoise CT images. Kumar and Diwakar [20] applied the Haar-type wavelet transform technique to denoise CT images. Chen et al. [21] applied a new denoising method for low-dose CT, along with the deep learning method. Dabov et al. [22] conceptualized a new image denoising strategy based on transform domains. They compared the experimental results that have been obtained through advanced algorithms. These algorithms are called block-matching and 3-D filtering (BM3D) [23].

In the study, images of $\mathrm{CT}$, which have been obtained using medical imaging techniques in order to facilitate the diagnosis and treatment of the patient in medicine, are 
utilized. The process of obtaining a CT image is the process in which the noises in CT images first appear. Each step in the process brings natural phenomena to the fluctuations and adds a random number to the true brightness value of this pixel. If it was possible to know which type of noise was occurring at which step, it could be done with a zero error. However, this is not possible in practice. So denoising in the images continues to be a major problem in the field of imaging. The image is restored in order to reduce or eliminate the data losses and distortions caused by noise during the formation of an image. The apparent loss of data is noise-induced and this loss is the deviation of the noisy pixel from the true pixel value. In this context, we worked on images of the CT that were obtained by using medical imaging techniques in order to facilitate the diagnosis and treatment of the patient in medicine. It is aimed to correctly determine and classify the noisy pixels of CT images by using ELM classification method.

\section{MATERIALS}

In this study, the input volumes were taken from the Stanford volume data archive [24]. The database comprises 99 slices of $256 \times 256$ pixels and 8-bit TIF files. The images were obtained using a CT device, and collected from different positions.

A 3-D surface model was constructed utilizing 2-D medical image data, which has been obtained from different locations in the CT device. Figurelillustrates a noisy 3-D surface model rendering from $\mathrm{CT}$ images in the database.

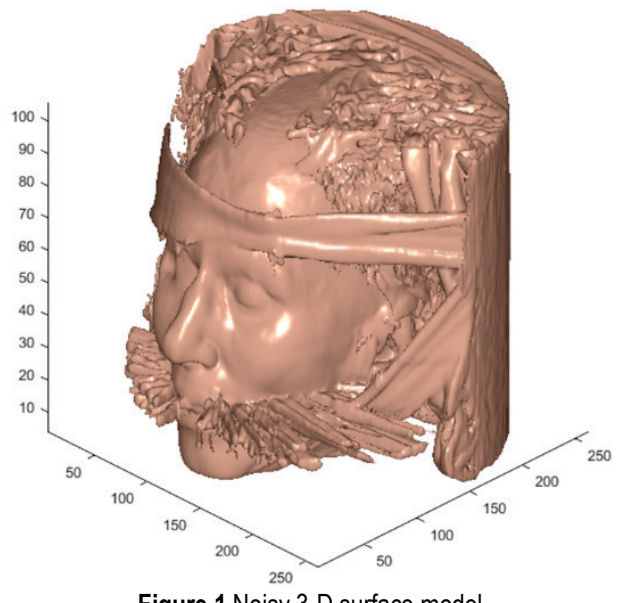

Figure 1 Noisy 3-D surface model

As observed in the 3-D surface model, the head of the cadaver appears to be covered in a material similar to the piece of cloth, the back is covered together with an object similar to pillow, and the teeth are kept together by ironlike objects.

\section{METHODS}

A 3-D surface model was created using 2-D medical image data taken from various positions of the CT machine. Subsequently, the model was tested using noise detection techniques, including ELM and several filtering techniques. The adjustment of contrast and brightness of each CT section was then performed utilizing noise detection and image processing techniques. After noting the similarities of 2-D images, the changing distance between the images was calculated by applying edge detection algorithms. The 3-D surface model was obtained by applying volume-rendering techniques.

The method of ELM was employed for training single hidden layer feed-forward Artificial Neural Networks (ANN). ELM method was used to determine noisy pixels of the images. Two datasets were created and tested through the ELM method, after performing the required classifications.

\subsection{Feature Extraction}

In this stage, first the noisy pixels were determined. Following this, the pixel values around the targeted noisy pixel were located. Each noisy pixel has 8 neighborhoods in its frame $\left(f_{n}\right)$ and 9 neighborhoods in its previous frame $\left(f_{n-1}\right)$ and 9 in the next frame $\left(f_{n+1}\right)$ (Figure 2$)$. For each pixel value in the reference image, 26 connected pixel values were examined ( 8 neighborhoods in its frame, 9 neighborhoods in its previous frame, and 9 neighborhoods in the next frame). The average weights of the noisy pixels were calculated through this model utilizing the similarity of pixel neighborhoods.

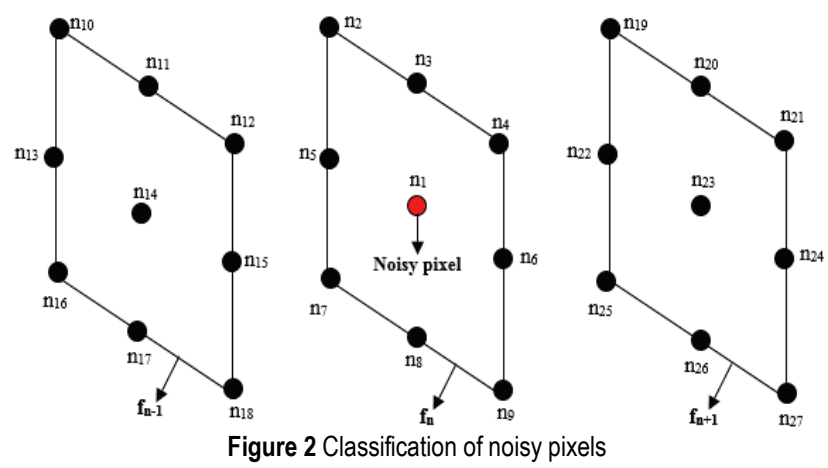

In order to generate a data set in the classification phase, each pixel determined as noisy by the ELM system was assigned as a value of 1 , and a value of 0 was assigned to a pixel that was noiseless (Table 1). The classification was done for the entire system as presented in Figure 2, taking into consideration neighborhood relations for each noisy pixel.

\begin{tabular}{cccc}
\multicolumn{4}{c}{ Table 1 Classification of noisy pixels } \\
\hline $\mathrm{f}_{\mathrm{n}-1}$ & $\mathrm{f}_{\mathrm{n}}$ & $\mathrm{f}_{\mathrm{n}+1}$ & Classification \\
\hline 9 & 8 & 9 & $1:$ Noisy Pixel \\
\cline { 2 - 3 } members & members & members & $\begin{array}{c}0: \text { Noiseless } \\
\text { Pixel }\end{array}$ \\
\hline
\end{tabular}

In order to create a data set, noisy pixels have to be determined. Eq. (1) presents the frame number $\left(f_{n}\right)$ and the pixel values $(x, y)$ for a noisy pixel sample $(I)$.

$$
I\left(f_{n} ; x, y\right)=\text { noise }
$$

In order to generate a data set in the classification phase, each pixel determined as noisy by the ELM system 
was assigned a value of 1 , and a value of 0 was assigned to a pixel that was noiseless (Tab. 1).

By performing these classifications, two datasets were created. During the first stage of the designed system, the dataset was created following each pixel belonging to the image being determined to either be noisy or not. To detect noisy pixels, the created dataset was trained by the ELM method and compared with other images. In the last stage, all the trained datasets were tested using cross-validation methods, and the overall performance values of the system were measured.

\subsection{Extreme Learning Machines}

The ELM was proposed to be applied in the single hidden layer feed-forward ANN. The method was suggested for use in the hidden resources decomposition [25]. In the ELM, input layer weights and threshold values are randomly assigned and the output layer weights are calculated analytically [26]. In the literature, the images are usually classified into hidden sources based on changes in weight [27]. This algorithm was applied only for single-hidden layer feed-forward ANN. Many researchers have reported the capability of feed-forward ANN in solving problems [28-30].

Consequently, the ELM has an ability of higher generalization $[25,31]$. As it can be evidently noted, there are no repeated steps in the training process. In many applications, good performances with high learning speeds can be attained [32]. The structure of the single hidden layer ANN has been visualized in Fig. 3. The output of the artificial neural network to be calculated is provided by Eq. (2).

$$
Y_{p}=\sum_{j=1}^{m} \beta_{j, k} g\left(\sum_{i=1}^{n} w_{i, j} x_{i}+b_{j}\right)
$$

Herein, $x_{1 \ldots n}, y_{1 \ldots p,} \beta_{1 \ldots m}, w_{1 \ldots n, 1 \ldots m}, b_{i \ldots m}$, and $g($. refer to input vectors, output vectors, output layer weights, connection weights between input, and hidden layers, threshold values, and activation functions respectively. The $w_{i, j}$ and bias are randomly assigned. According to the calculating scheme of weights and biases in ELM, its training stage is rapid and the generalization capacity is high [32].

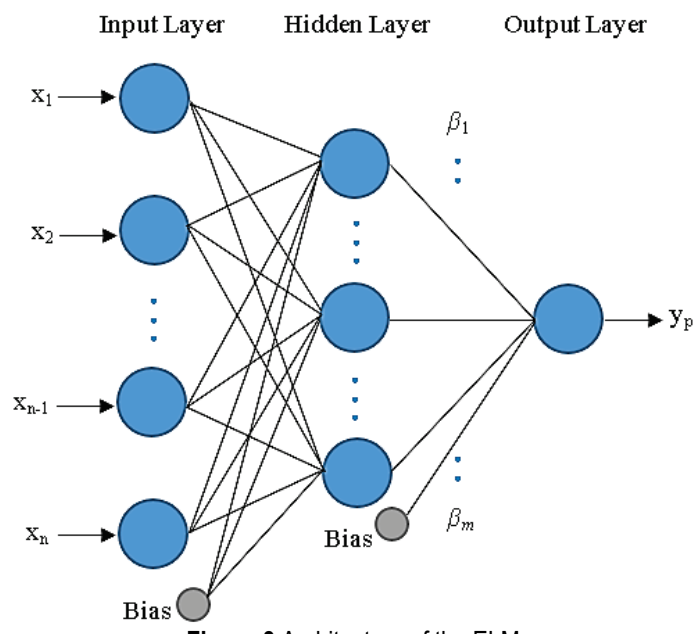

Figure 3 Architecture of the ELM
When training of the ELM has been initialized, the input weight and bias values are initialized with random values selected in the interval $(0,1)$. Thus, the ELM functions very fast by minimizing the training error and the output weights, with a single matrix inversion, without the need to fix any parameters.

\subsection{Error Metrics}

The error metrics, mean squared error (MSE) and peak signal-to-noise ratio $(P S N R)$ are used to draw comparisons between varied imaging methods. $M S E$ is the cumulative squared error between the original image and the distorted image. The PSNR is an index that reveals radiometric errors in the produced enriched image. The distinction between the processed and original image can be analysed by means of a certain parameter such as $P S N R$. This parameter will determine the quality of the processed image in relation to the original image. PSNR is a similarity measure utilized for comparing images [33]. It is defined in terms of $M S E$ and used for image compression, denoising, deblurring, and enhancement to a certain extent. It is also applied to quantify the level of similarity or agreement between two datasets. The first equation in the PSNR calculation is used to find the MSE (Eq. 3) [34].

$M S E=\frac{1}{M N} \sum_{x=1}^{M} \sum_{y=1}^{N}\left(I(x, y)-I^{\prime}(x, y)\right)^{2}$

The PSNR parameter is calculated to determine the quality of the output image that has been obtained by the spectral subtraction method and the image quality can be increased (Eq. (4)).

$P S N R=20 \cdot \log _{10}\left(\frac{L}{\sqrt{M S E}}\right)$

In Eq. (3) and Eq. (4), $I(x, y)$ is the pixel value of the original image; $I^{\prime}(x, y)$ is the pixel value of the distorted image; $M$ and $N$ are the number of pixel values from 0 to 255 for 8 bit images; and $L$ (Peak) refers to the highest (255) possible pixel value [35]. The PSNR is measured in logarithmic decibels $(\mathrm{dB})$.

Since a lower value for $M S E$ implies lesser errors, the error decreases in the image as the value of the MSE decreases. Moreover, a low value of MSE means a high value of $P S N R$, as can be noted from the inverse relationship between MSE and PSNR. For this reason, the value of low MSE or high PSNR generates better results.

\section{RESULTS AND DISCUSSIONS 4.1 Obtained Noise Detection Results by ELM}

In this study, the ELM method was used to determine noisy pixels. First, the dataset was divided into five equal parts (Fig. 4), subsequently, four of them were considered as training data, and the remaining data was utilized as test data. The training and classification process was carried out five times through cross validation, whereby different dataset was used each time. As a result of the 
process, the average of the accuracy values obtained in each fold was taken. The results give the accuracy of the classification algorithm.

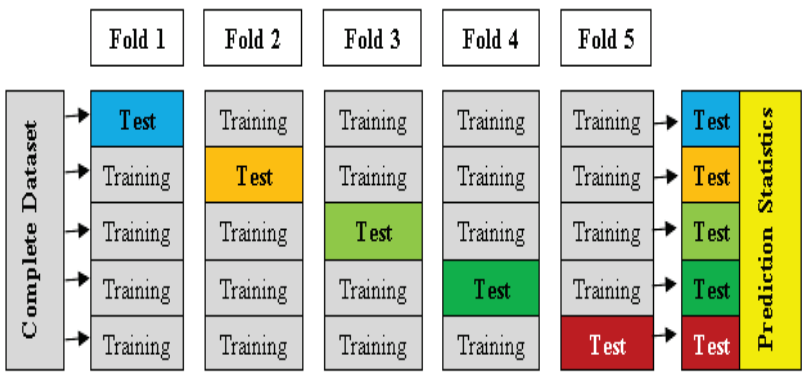

Figure 4 Estimated statistics with $\mathrm{k}$-fold cross-validation $(k=5)$

The optimum success rate of the system was calculated using the ELM method by varying the number of neurons in the hidden layer during training, and testing. Sigmoid, Sine, Hard Limit, Triangular Basis, and Radial Basis were considered as the activation function. The number of neurons in the hidden layer was considered between 10 and 1500 . Therefore, the number of neurons in the most appropriate hidden layer was varied from 10 to 1500 with different neuron steps. Following the achievement of the optimum success rate, the optimum number of neurons was determined at the point where the performance of the system decreased. It was observed that the best success rate of the system was attained through the Sigmoid activation function. The test success rate of the system was between $75.37-87.20 \%$ (Tab. 2). The highest success rate was achieved in the hidden layer of 250 neurons.

Table 2 Classification of noisy pixels

\begin{tabular}{|c|c|c|c|c|c|}
\hline \multirow{2}{*}{$\begin{array}{l}\text { Number of } \\
\text { Neurons in } \\
\text { the Hidden } \\
\text { Layer }\end{array}$} & \multicolumn{5}{|c|}{ Activation Function } \\
\hline & Sigmoid & Sine & $\begin{array}{l}\text { Triangular } \\
\text { Basis }\end{array}$ & $\begin{array}{c}\text { Radial } \\
\text { Basis }\end{array}$ & $\begin{array}{l}\text { Hard } \\
\text { Limit }\end{array}$ \\
\hline 10 & 75.37 & 63.42 & 74.98 & 74.68 & 75.17 \\
\hline 25 & 77.23 & 63.80 & 74.92 & 74.57 & 76.36 \\
\hline 50 & 79.70 & 63.88 & 75.22 & 75.98 & 78.28 \\
\hline 75 & 81.32 & 64.05 & 76.33 & 76.28 & 80.16 \\
\hline 100 & 84.53 & 65.87 & 77.65 & 77.46 & 83.15 \\
\hline 150 & 86.35 & 65.52 & 77.82 & 78.32 & 84.49 \\
\hline 200 & 87.18 & 66.28 & 78.41 & 79.67 & 85.39 \\
\hline 250 & 87.20 & 66.08 & 77.98 & 79.18 & 85.52 \\
\hline 500 & 85.59 & 65.75 & 77.36 & 77.32 & 83.85 \\
\hline 750 & 84.53 & 65.87 & 77.65 & 77.46 & 83.15 \\
\hline 1000 & 83.72 & 64.80 & 77.39 & 75.54 & 82.16 \\
\hline 1500 & 82.19 & 64.32 & 75.77 & 75.81 & 80.24 \\
\hline
\end{tabular}

It has been observed that the test success rate decreased when the number of neurons exceeded a certain level. This is due to the fact that the learning algorithm, along with the increase in the number of neurons, memorizes the system and cannot decide when it encounters a new situation. The classification algorithm that memorizes the system due to the overfitting situation will have difficulty in deciding whether the pixels are noisy or denoised. In the event of overfitting, the ELM algorithm diverts from solving the main problem and focuses on keeping the values given to it. Since increasing the number of neurons results in negative effects on the system, it is necessary to keep the number of neurons at an optimum level.
Results of classification system were calculated by some commonly used statistical measures such as MSE and PSNR. A low MSE value means lesser error. When the $M S E$ value decreases, the error rate in the image also decreases. There is an inverse ratio between $M S E$ and $P S N R$ values. Namely, a low value of $M S E$ implies a high value of $P S N R$. Moreover, it can be clearly viewed from the MSE and PSNR results presented in Tab. 3 that the most successful performance value is reached with the number of hidden neurons at 250 . The best result of the system was noted with the MSE value of 0.148 and the PSNR value of 56.46 .

Table 3 The effect of changing the number of neurons in the hidden layer on the algorithm performance

\begin{tabular}{|c|c|c|}
\hline $\begin{array}{c}\text { Number of Neurons in the } \\
\text { Hidden Layer }\end{array}$ & MSE & PSNR \\
\hline 10 & 0.266 & 53.911 \\
\hline 25 & 0.248 & 54.225 \\
\hline 50 & 0.223 & 54.681 \\
\hline 75 & 0.207 & 55.010 \\
\hline 100 & 0.175 & 55.742 \\
\hline 150 & 0.156 & 56.221 \\
\hline 200 & 0.148 & 56.458 \\
\hline $\mathbf{2 5 0}$ & $\mathbf{0 . 1 4 8}$ & $\mathbf{5 6 . 4 6 0}$ \\
\hline 500 & 0.164 & 56.013 \\
\hline 750 & 0.175 & 55.742 \\
\hline 1000 & 0.183 & 55.550 \\
\hline 1500 & 0.198 & 55.200 \\
\hline
\end{tabular}

The noise pixel detection of the system was compared with the other images in the CT database, and the noisy pixels were accurately marked. The noises obtained from the images of different segments are displayed in Fig. 5. These figures show that the ELM method is successful in detecting intense noisy pixels on the outer side of images, with low noise on the inside.

\subsection{The Results of the Traditional Filters}

Some segmentation processes were performed to eliminate the noise in the data. Additionally, filtering algorithms were applied to obtain the required contour areas. Gaussian, Kuwahara, and NLMF filters were tested for the most intense 2-D noisy images.

The Gaussian filters use images with a Gaussiansmoothing kernel specified by sigma. The sigma values range between 0.5 (default) and 2 . The sigma variant is used for the standard deviation of the Gaussian kernel. The results for different sigma values 0.5 , and 1.5 , of the Gaussian filter are presented in Fig. 6. As the value of sigma increases, the required locations on the image become blurred.

The principle of NLMF is as follows: A local pixel region, surrounding a given pixel, is compared to the pixel patches in the neighborhood. The central pixels of the patches are averaged, depending on the quadratic pixel distance between the patches. The strength of the filter is considered an adaptively varying coefficient of the NLMF. The filter strength default value is approximately 0.05 . The result for different strength values of 0.05 and 0.2 of the NLMF has been displayed in Fig. 7. As the strength value increases, areas that fall outside the noisy region become blurred. 


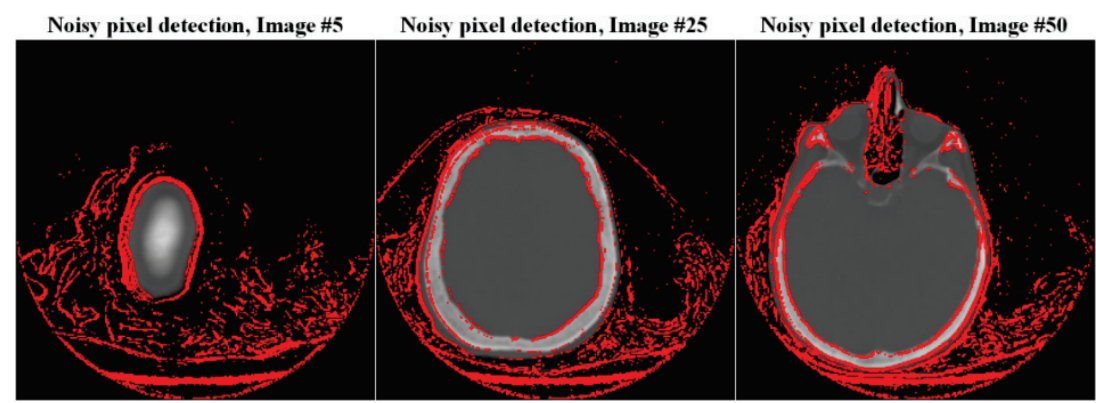

Noisy pixel detection, Image \#75

Noisy pixel detection, Image \#76

Noisy pixel detection, Image \#77
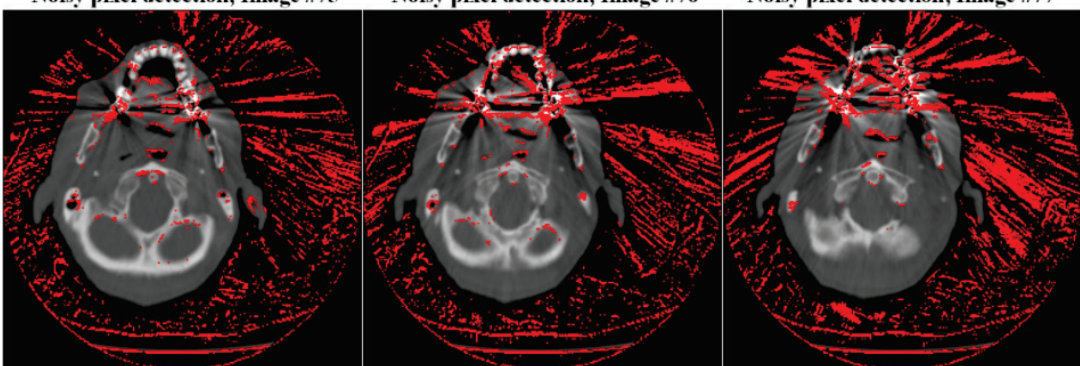

Figure 5 Noisy pixel detection with ELM method in some images

Gaussian \#75, sigma $=0.5$ Gaussian \#76, sigma $=0.5$

Gaussian \#77, sigma= 0.5
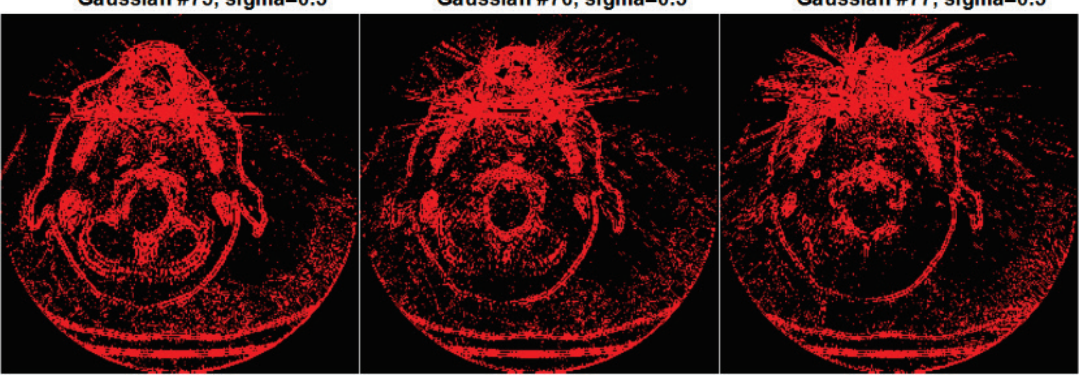

Gaussian \#75, sigma=1.5

Gaussian \#76, sigma=1.5

Gaussian \#77, sigma=1.5
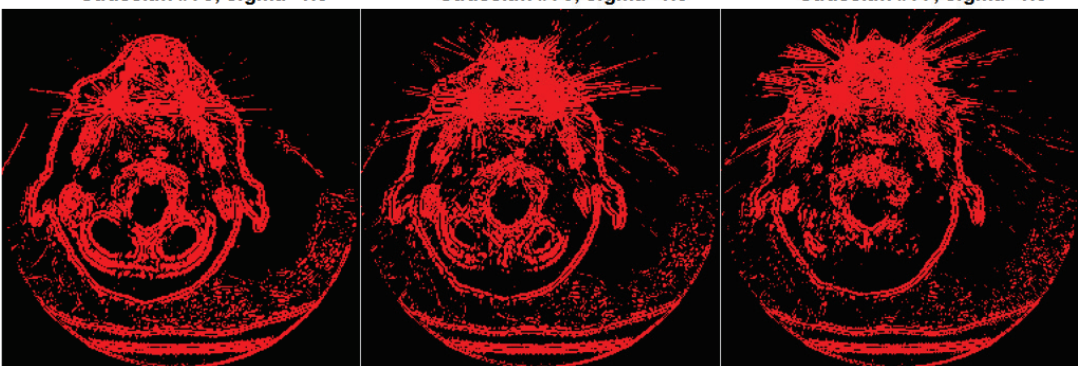

Figure 6 The results for different sigma values of the Gaussian filter

NLMF \#75, strength $=0.05$

NLMF \#76, strength $=0.05$

NLMF \#77, strength $=0.05$
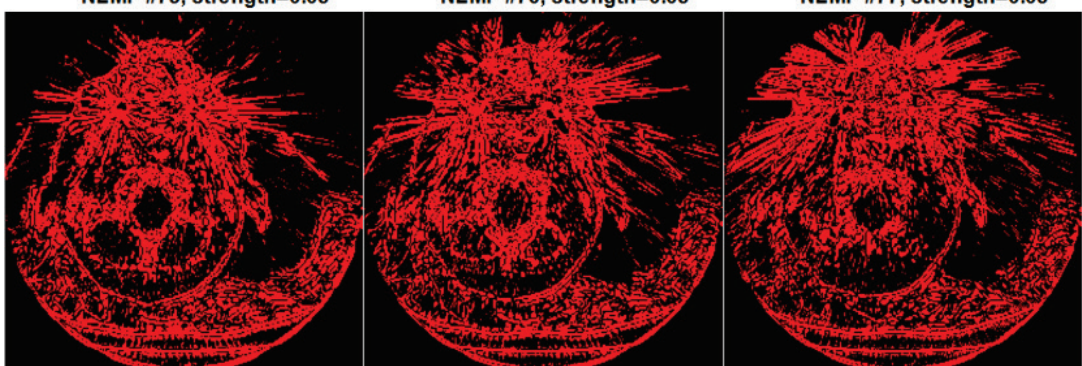

NLMF \#75, strength $=0.2$

NLMF \#76, strength $=0.2$

NLMF \#77, strength $=0.2$
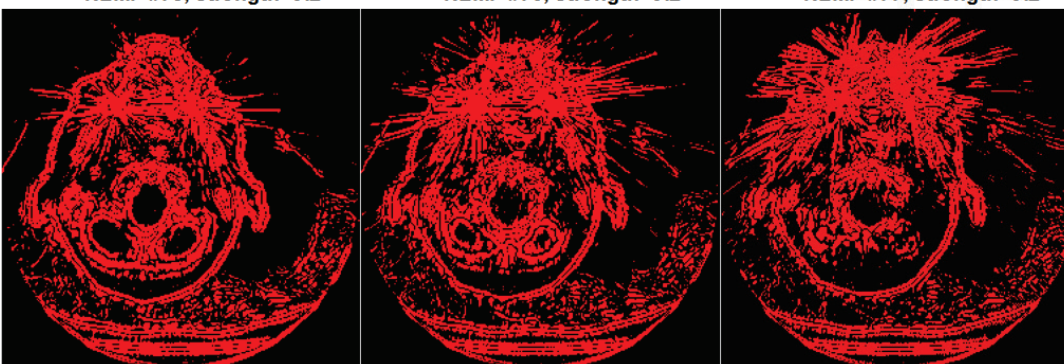

Figure 7 The results for different strength values of the NLMF 
The Kuwahara filter uses a floating windows size approach to access each pixel in the image. The size of the window is preselected and can be changed depending on the desired level of blur. The default value for window size is 5 . The results of different window size values of the Kuwahara filter are provided in Fig. 8. The window size in the Kuwahara filter is compared for different images with values 3 and 5 .

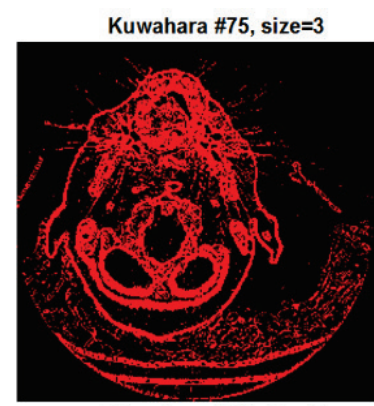

Kuwahara $\# 75$, size $=5$

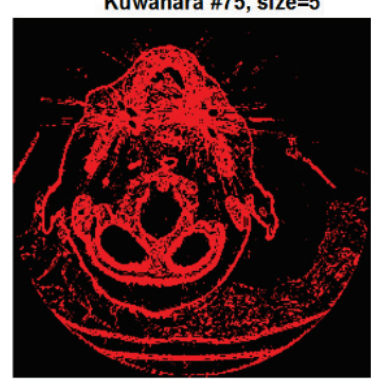

Figure 8 The results of different window size values of the Kuwahara filter
Kuwahara \#77, size=3

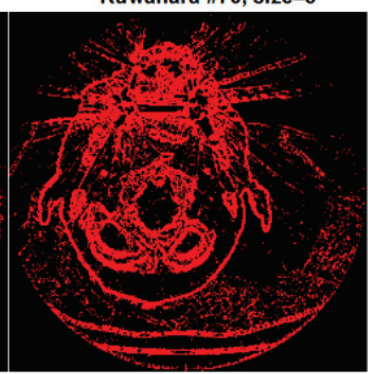

Kuwahara \#76, size $=5$

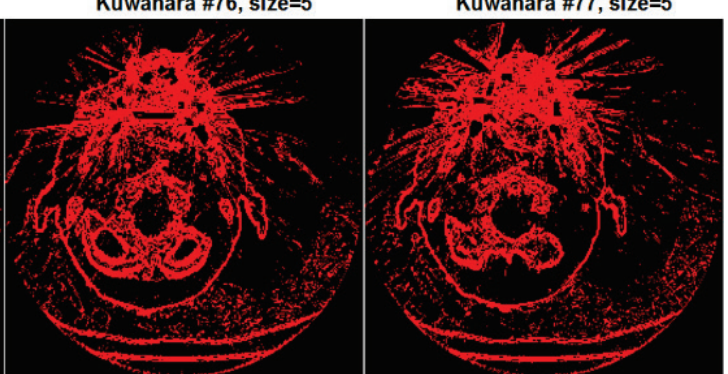

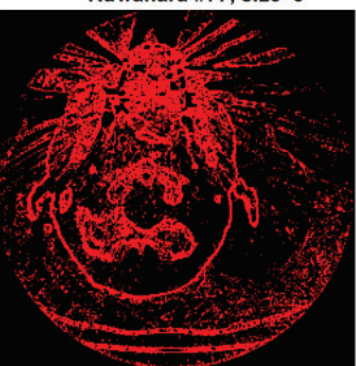

Kuwahara $\# 77$, size $=5$

\subsection{The Comparisons}

In the CT images used, by applying the results to all the pixels in the image, the traditional filtering methods may result in certain image data being lost, relevant to the medical field. The results of widely-used Gaussian, Kuwahara and NLMF filters were tested and compared in terms of the filtering method. The results of ELM and filters are compared and visualized for the noisiest $75^{\text {th }}$, $76^{\text {th }}$, and $77^{\text {th }} \mathrm{CT}$ images in the database.

Table 4 Accuracy (\%) values of the traditional filters and ELM methods

\begin{tabular}{|c|c|c|c|c|}
\hline Method & Image 75 & Image 76 & Image 77 & $\begin{array}{c}\text { Overall System } \\
\text { Performance }\end{array}$ \\
\hline $\begin{array}{c}\text { Gaussian } \\
\text { Filters }\end{array}$ & 74.79 & 73.14 & 72.49 & 77.73 \\
\hline $\begin{array}{c}\text { Kuwahara } \\
\text { Filters }\end{array}$ & 77.51 & 76.59 & 74.97 & 79.17 \\
\hline $\begin{array}{c}\text { NLM } \\
\text { Filters }\end{array}$ & 67.46 & 66.38 & 65.36 & 72.92 \\
\hline ELM & 84.45 & 83.78 & 81.74 & 87.20 \\
\hline
\end{tabular}

Each pixel value in the images has their individual importance since it is difficult and sensitive to examine the images in the medical region. The methods used to reduce noise in medical images may damage the remaining pixels. Therefore, the pixels in the CT images were individually examined with a pixel-based approach. The results of the ELM method were used to determine noisy pixels for the CT images, which are shown in Fig. 5. As presented in the figure, noisy pixel detection through other methods was applied to the entire image. However, our goal was also to not damage the other pixels while trying to detect noisy pixels in the images. The ELM method has performed the local noise detection, and that has provided successful results in detecting noisy pixels. The results of the accuracy ratios to determine noisy pixels of Gaussian, Kuwahara, and NLMF filters and the ELM method are given in Tab. 4. The success rates of the noisy pixel detections filters taken as an example in the table and the overall system performance of the ELM method are seen.

Considering the literature review, some basic approaches for noise pixel estimation in the images are outlined in Tab. 5. It has been observed that the overall accuracy of the proposed system provides good results compared to other approaches in the literature.

Table 5 Algorithm performance comparison against image denoising of CT

\begin{tabular}{|c|c|c|c|}
\hline Reference & Dataset & Method & $P S N R$ \\
\hline $\begin{array}{c}\text { Proposed } \\
\text { Method }\end{array}$ & Stanford CT Head & ELM & 56.46 \\
\hline$[13]$ & $\begin{array}{l}\text { IBSR and IDEA GROUP } \\
\text { Dataset }\end{array}$ & HLWF & 45.85 \\
\hline$[15]$ & $\begin{array}{c}\text { ELCAP Public Lung Image } \\
\text { Database }\end{array}$ & $\begin{array}{c}\text { Wavelet } \\
\text { Transform }\end{array}$ & 27.12 \\
\hline$[16]$ & BrainWeb: Simulated Brain & CNLM & 37.18 \\
\hline$[17]$ & BrainWeb: Simulated Brain & PSNLM & 37.60 \\
\hline$[18]$ & $\begin{array}{c}\text { Japanese Society of } \\
\text { Radiological Technology } \\
\text { (JSRT) }\end{array}$ & $\begin{array}{c}\text { Deep feed } \\
\text { forward CNN }\end{array}$ & 39.25 \\
\hline [19] & $\begin{array}{c}\text { Cadaver CT images (collected } \\
\text { at Massachusetts General } \\
\text { Hospital) }\end{array}$ & $\mathrm{CNN}$ & 31.12 \\
\hline$[20]$ & $\begin{array}{c}\text { ELCAP Public Lung Image } \\
\text { Database }\end{array}$ & $\begin{array}{l}\text { Haar-type } \\
\text { wavelet } \\
\text { transform } \\
\end{array}$ & 32.87 \\
\hline$[21]$ & $\begin{array}{l}\text { The Cancer Imaging Archive } \\
\text { (TCIA) }\end{array}$ & Deep CNN & 42.42 \\
\hline [22] & Database of natural images & BM3D & 32.27 \\
\hline \multirow{2}{*}[36]{} & Stanford CT dataset & \multirow{2}{*}{ Ray-leaping } & 52.03 \\
\hline & Visible woman CT dataset & & 51.41 \\
\hline
\end{tabular}

The higher the PSNR value, the better the restoration of the degraded image according to the original image. The PSNR value is higher for evenly distributed modifications than sparsely distributed modifications. Thus, instead of modifying one pixel value by a large 
amount, modifying many pixels by very small amounts will result in higher PSNR. If the PSNR value is high, the $M S E$ between the original image and reconstructed image is very low. This implies that the image has been properly restored. Following the same logic, the restored image quality is better.

According to the maximum signal value of the images, it is necessary to reduce the maximum value of the MSE between the images. In other words, the MSE value is attempted to tend towards zero. If the $M S E$ value is calculated with two identical images, the value becomes zero and the PSNR value will be undefined. The $M S E$ being zero implies no noise is present in the image.

The actual PSNR value may not be relevant, but the comparisons between the images' reconstructed values offer a measure of quality. The PSNR is an error metric utilized to compare changes in the original image and the restored image. However, the comparison of the PSNR value for different images is meaningless. For example, an image with a $P S N R$ value of $25 \mathrm{~dB}$ may look better than another image with a $P S N R$ value of $35 \mathrm{~dB}$. The corresponding PSNR metric for acceptable images for use in digital radiology varies from $40 \mathrm{~dB}$ to $50 \mathrm{~dB}$ depending on the metric used [36]. In comparing an original picture and a coded picture, the PSNR figures typically range from +25 to $+35 \mathrm{~dB}$ [37]. In steganography images, the best PSNR result varies from 50 to $55 \mathrm{~dB}$ [38].

\section{CONCLUSION}

The purpose of this study was to increase the quality of outputs produced by medical imaging systems. Primarily, the methods and approaches adopted in 2-D were applied to 3-D with different aspects. In this context, several common noise detection techniques that are widely used in 2-D imaging were investigated, including Gaussian, Kuwahara, and NLMF filters. These common filtering techniques were used for noise detection in CT images. However, it was noted that pixel values in noiseless regions also changed, even though each pixel value has individual importance in diagnosis of medical regions. Thus, other pixel values should not be altered during the denoising process. The ELM method was successfully applied to detect noisy pixels in CT images. It should be noted that, contrary to the other common methods, the ELM method achieves local noisy pixel detection. The performance of the system in detecting noisy pixel values was also provided. The test results concluded that this method yielded an efficient performance in the detection of noisy pixels. The experimental results indicated that the ELM method provides better success rates in the detection of noisy pixels.

\section{REFERENCES}

[1] Yagel, R. \& Kaufman, A. E. (1992). Template-Based Volume Viewing. Computer Graphics Forum, 11(3), 153157. https://doi.org/10.1111/1467-8659.1130153

[2] Goyal, G., Bansal, A. K., \& Singhal. M. (2012). Comparison of Denoising Filters on Greyscale TEM Image for Different Noise. IOSR Journal of Computer Engineering (IOSRJCE), 5(4), 37-44. https://doi.org/10.9790/0661-0543744
[3] Pham, D. L., Xu, C., \& Prince, J. L. (2000). Current methods in medical image segmentation. Annual review of biomedical engineering, 2(1), 315-337. https://doi.org/10.1146/annurev.bioeng.2.1.315

[4] Yan, C., Xie, H., Liu, S., Yin, J., Zhang, Y., \& Dai, Q. (2017). Effective Uyghur Language Text Detection in Complex Background Images for Traffic Prompt Identification. IEEE Transactions on Intelligent Transportation Systems, 99, 1-10. https://doi.org/10.1109/TITS.2017.2749977

[5] Buades, A., Coll, B., \& Morel, J. M. (2005). A non-local algorithm for image denoising. Computer Vision and Pattern Recognition, CVPR 2005, IEEE Computer Society Conference on., 60-65. https://doi.org/10.1109/CVPR.2005.38

[6] Bansal, M., Devi, M., Jain, N., \& Kukreja, C. (2014). A proposed approach for biomedical image denoising using PCA_NLM. International Journal of Bio-Science and BioTechnology, 6(6), 13-20. https://doi.org/10.14257/ijbsbt.2014.6.6.02

[7] Mukherjee, P. S. \& Qiu, P. (2011). 3-D Image Denoising by Local Smoothing and Nonparametric Regression. Technometrics, 53, 196-208. https://doi.org/10.1198/TECH.2011.10070

[8] Coupé, P., Yger, P., Prima, S., Hellier, P., Kervrann, C., \& Barillot, C. (2008). An optimized blockwise nonlocal means denoising filter for 3-D magnetic resonance images. IEEE transactions on medical imaging, 27(4), 425-441. https://doi.org/10.1109/TMl.2007.906087

[9] Buades, A., Bartomeul, C., \& Morel, J. M. (2005). A review of image denoising algorithms, with a new one. Multiscale Modeling \& Simulation, 4(2), 490-530. https://doi.org/10.1137/040616024

[10] Efros, A. A. \& Leung, T. K. (1999). Texture synthesis by non-parametric sampling. Computer Vision, The Proceedings of the Seventh IEEE International Conference on, 1033-1038 https://doi.org/10.1109/ICCV.1999.790383

[11] Weijer, J. \& Boomgaard, R. (2001). Local Mode Filtering. Proceedings of the IEEE Computer Society Conference on Computer Vision and Pattern Recognition, 428-433. https://doi.org/10.1109/CVPR.2001.990993

[12] Suganthi, P. D. M. (2014). Performance Evaluation of Various Denoising Filters for Medical Image. International Journal of Computer Science and Information Technologies, 5(3), 4205-4209.

[13] Kalavathi, P. \& Priya, T. (2016). Removal of impulse noise using Histogram-based Localized Wiener Filter for MR brain image restoration. IEEE International Conference on Advances in Computer Applications, 4-8. https://doi.org/10.1109/ICACA.2016.7887913

[14] Bhadauria, H. S. \& Singh, A. (2013). Wavelet and Canny Based Edge Detection Method for Noisy Lung CT Image. International Journal of Emerging Technology and Advanced Engineering, 3, 776-780.

[15] Gabralla, L., Mahersia, H., \& Zaroug, M. (2015). Denoising CT Images using wavelet transform. International Journal of Advanced Computer Science and Applications (IJACSA), 6, 125-129. https://doi.org/10.14569/IJACSA.2015.060520

[16] Chen, G., Zhang, P., Wu, Y., Shen, D., \& Yap, P. T. (2016). Denoising magnetic resonance images using collaborative non-local means. Neurocomputing, 177, 215227. https://doi.org/10.1016/j.neucom.2015.11.031

[17] Yang, J., Fan, J., Ai, D., Zhou, S., Tang, S., \& Wang, Y. (2015). Brain MR image denoising for Rician noise using pre-smooth non-local means filter. Biomedical engineering online, 14(2), 1-20. https://doi.org/10.1186/1475-925X-14-2

[18] Jifara, W., Jiang, F., Rho, S., Cheng, M., \& Liu, S. (2017). Medical image denoising using convolutional neural 
network: a residual learning approach. The Journal of Supercomputing, 1-15. https://doi.org/10.1007/s11227-017-2080-0

[19] Yang, Q., Yan, P., Kalra, M. K., \& Wang, G. (2017). CT Image Denoising with Perceptive Deep Neural Networks. The $14^{\text {th }}$ International Meeting on Fully ThreeDimensional Image Reconstruction in Radiology and Nuclear Medicine, 858-863. https://doi.org/10.12059/Fully3D.2017-11-3202015

[20] Kumar, M. \& Diwakar, M. (2018). CT image denoising using locally adaptive shrinkage rule in tetrolet domain. Journal of King Saud University-Computer and Information Sciences, 30, 41-50. https://doi.org/10.1016/j.jksuci.2016.03.003

[21] Chen, H., Zhang, Y., Zhang, W., Liao, P., Li, K., Zhou, J., \& Wang, G. (2017). Low-dose CT via convolutional neural network. Biomedical optics express, 8, 679-694. https://doi.org/10.1364/BOE.8.000679

[22] Dabov, K., Foi, A., Katkovnik, V., \& Egiazarian, K. (2007). Image denoising by sparse 3-D transform-domain collaborative filtering. IEEE Transactions on image processing, 16, 2080-2095. https://doi.org/10.1109/TIP.2007.901238

[23] Shah, M. \& Dalal, U. D. (2014). 3D-image restoration technique using Genetic Algorithm to solve blurring problems of images. The Imaging Science Journal, 62(7), pp. 365-374. https://doi.org/10.1179/1743131X13Y.0000000067

[24] Levoy, M. (2002). The Stanford volume data archive CT Head Dataset. Stanford Volume Graphics Laboratory, available from http://graphics.stanford.edu/data/voldata/

[25] Huang, G. B., Zhu, Q. Y., \& Siew, C. K. (2006). Extreme learning machine: Theory and applications. Neurocomputing, 70(1), 489-501. https://doi.org/10.1016/j.neucom.2005.12.126

[26] Özerdem, M. S., Acar, E., \& Ekinci, R. (2017). Soil Moisture Estimation over Vegetated Agricultural Areas: Tigris Basin, Turkey from Radarsat-2 Data by Polarimetric Decomposition Models and a Generalized Regression Neural Network. Remote Sensing, 9(4), 395. https://doi.org/10.3390/rs9040395

[27] Tan, Y., Wang, J., \& Zurada, J. M. (2001). Nonlinear blind source separation using a radial basis function network. IEEE Transactions on Neural Networks, 12(1), 124-134. https://doi.org/10.1109/72.896801

[28] Cybenko, G. (1989). Approximation by superpositions of a sigmoidal function. Mathematics of Control, Signals, and Systems (MCSS), 2(4), 303-314. https://doi.org/10.1007/BF02551274

[29] Funahashi, K. I. (1989). On the approximate realization of continuous mappings by neural networks. Neural networks, 2(3), 183-192. https://doi.org/10.1016/0893-6080(89)90003-8

[30] Hornik, K., Stinchcombe, M., White, H. (1989). Multilayer feedforward networks are universal approximators. Neural networks, 2(5), 359-366. https://doi.org/10.1016/0893-6080(89)90020-8

[31] Ertuğrul, Ö. F. \& Kaya, Y. (2014). A Detailed Analysis on Extreme Learning Machine and Novel Approaches Based on ELM. American Journal of Computer Science and Engineering, 1(5), 43-50.

[32] Tang, J., Deng, C., \& Huang, G. B. (2016). Extreme learning machine for multilayer perceptron. IEEE transactions on neural networks and learning systems, 27(4), 809-821. https://doi.org/10.1109/TNNLS.2015.2424995

[33] Wang, Y., Fu, J., Adhami, R., \& Dihn, H. (2016). A novel learning-based switching median filter for suppression of impulse noise in highly corrupted colour images. The Imaging Science Journal, 64(1), pp. 15-25. https://doi.org/10.1080/13682199.2015.1104068
[34] Hanafy, M. A. (2017). MRI Medical Image Denoising by Fundamental Filters. SCIREA Journal of Computer, 2(1), 12-26. https://doi.org/10.5772/intechopen.72427

[35] Zhou, Y., Zeng, F. Z., \& Yang, G. F. (2012). The research for tamper forensics on MPEG-2 video based on compressed sensing. Machine Learning and Cybernetics (ICMLC), 2012 International Conference on, 1080-1084. https://doi.org/10.1109/ICMLC.2012.6359505

[36] Çelebi, Ö. C. \& Çevik, U. (2010). Accelerating volume rendering by ray leaping with back steps. Computer methods and programs in biomedicine, 97(2), 99-113. https://doi.org/10.1016/j.cmpb.2009.05.007

[37] Singh, H. \& Neeru, N. (2013). Performance Improvement of Decision Based Un-symmetric Trimmed Midpoint Filter for Removing High Density Salt and Pepper Noise. International Journal of Emerging Trends \& Technology in Computer Science, 2(3), 254-257.

[38] Jassim, F. A. (2013). A novel steganography algorithm for hiding text in image using five modulus method. International Journal of Computer Applications, 72(17), 39-44. https://doi.org/10.5120/12637-9448

\section{Contact information}

Abidin ÇALIŞKAN, PhD

(Corresponding author)

Department of Computer Engineering,

Batman University, Batman, Turkey

E-mail: abidin.caliskan@batman.edu.tr

\section{Ulus ÇEVIK, Professor}

Department of Electrical and Electronics Engineering,

Çukurova University, Adana, Turkey

E-mail: ucevik@cu.edu.tr 